Temporal evolution of a circular membrane subject to various boundary conditions

This article has been downloaded from IOPscience. Please scroll down to see the full text article.

2005 Eur. J. Phys. 26107

(http://iopscience.iop.org/0143-0807/26/1/012)

View the table of contents for this issue, or go to the journal homepage for more

Download details:

IP Address: 150.203.38.140

The article was downloaded on 26/09/2011 at 09:11

Please note that terms and conditions apply. 


\title{
Temporal evolution of a circular membrane subject to various boundary conditions
}

\author{
L K Castelano, M S Barbosa and D M Redondo \\ Instituto de Física de São Carlos, Universidade de São Paulo, 13560-970, São Carlos, SP, Brazil
}

Received 17 October 2004, in final form 18 October 2004

Published 14 December 2004

Online at stacks.iop.org/EJP/26/107

\begin{abstract}
In this paper we illustrate a standard problem of mathematical physics, by exploring the potential of using a computer algebra system in a classroom experiment. Although many textbooks describe the problem and solve its particular examples to variable extent, to the student is always left the task of imagining, after a few tedious calculations, the temporal evolution of the system. The aim of this paper is, using algebraic computation, to give a complete route to the problem of an asymmetrically perturbed circular membrane in viscous media with the usual boundary conditions, including the computer animation of the results.
\end{abstract}

\section{Introduction}

We have witnessed a steady increase in the availability of computers with sufficient CPU power to process numerical applications of physical and engineering interest that used to drain mainframe computer system resources a decade ago. The situation has improved more dramatically with the evolution of algebraic computational platforms to such a mature level that many theoretical studies are now done relying on these platforms and extending them concomitantly. The classroom reality has, with relatively few exceptions, overlooked such powerful tools and machinery, leading interested science students to explore these possibilities unsystematically by themselves. In the best case they can achieve some degree of comparison with the theory presented in classical texts, which is invariably limited to simple cases. It is the belief of the authors of this paper that such availability of tools should push us ahead towards giving a more complete view as possible so as to preserve the amount of intellectual effort a student should put in his/her academic formation.

The problem of the vibrations of a tense circular membrane, with a fixed border, is a classic problem of mathematical physics, having been solved for the first time close to the middle of the eighteenth century, by Euler [1, 2]. The solution envisaged by Euler made use 
of a power series that we today refer to as $J_{0}(x)$-the Bessel function of the first kind, which was to be formally introduced in 1824 by Bessel [3], a near sixty year interim.

In musical instruments employing circular membranes, such as the drum for example, the vibrations propagating on the membrane are of such a minute amplitude and much too fast to reveal visually the complex composition of normal modes. The use of a computer in this situation can be very welcome, especially considering the graphic animation facilities of systems such as (Maple, Mathematica, etc). In [4] one can see the use of such an environment, but restricted to normal modes and only of symmetrically driven membranes under fixed boundary conditions.

As will be reported in this paper, for the case of circular membranes and for many other vibrational problems, not only the full structure of normal modes at various boundary conditions but also the actual solution in time of an asymmetrically driven membrane can be analytically handled and graphically explored with relatively little effort by using such an algebraic environment and widespread available hardware.

In the majority of textbooks of mathematical physics, including some classics [5, 6], it is rare to see treated the boundary value problem of vibrations, induced by a out-of-centre perturbation, across a membrane with a circular hole concentric with its border. It is also rare to come across the solution of the analogue problem of undulations of the surface of a liquid in a circular reservoir. One can find a description of both situations without the asymmetric perturbation in the books [7-9]. These are both relevant educational problems in mathematical physics and are treated in the present paper with the aim of being a pedagogical contribution for the teaching of these topics.

Here, we collected most of the mathematics involved in the solution of cylindrically symmetric linear differential equations by illustrating the composition of eigenmodes of the circular membrane. Damping was added as it requires little more in terms of a tidy presentation of formulae, as opposed to a driven time dependent force and the important concept of resonance, which are left aside, as they would complicate the exposition and could be modelled alone in a much simpler application.

This paper starts by presenting a review of the basic procedure of the spectral decomposition and temporal evolution of separable differential equations. We then present three important boundary situations that this membrane could satisfy and present the graphics of eigenmodes and temporal evolution. It is important to remark that to fully accomplish the purpose of this paper the reader should be able to reproduce in his/her computer this simulation in detail as the visualization can only be partially printed.

\section{Solutions of the damping wave equation in cylindrical coordinates}

The sound of the drum comes from the oscillatory movement of the circular membrane fixed at the border. To describe a drum mathematically, we assume that the circular membrane is uniformly distended in all directions by a tension $T$, vibrating in the upward $z$-direction in a viscous medium with a small damping factor $\gamma$ and with no external forces. Under these considerations such a system follows the equation

$$
\nabla^{2} z(r, \theta, t)=\frac{1}{c^{2}} \frac{\partial^{2} z(r, \theta, t)}{\partial t^{2}}+\frac{\gamma}{c^{2}} \frac{\partial z(r, \theta, t)}{\partial t},
$$

where $c^{2}=T / \rho$ and $\rho$ is the mass density. The damping was included to give a more accurate description of a realistic problem. The same equation is obtained for a liquid having a free surface in the limit of small oscillations, under gravity and with a viscosity linearly dependent 
on velocity [7]. In order to complete the mathematical description we have to specify the boundary conditions, representing the physical situation we want to describe. In this work we adopt three different boundary conditions for the circular membrane.

The first problem we want to treat is the drum, which consists of a circular membrane with a fixed border, meaning that $z(r=R)=0$ for all $t$ and $\theta$, where $R$ is the radius of the membrane. The second physical situation is a membrane that has permitted movement at its contour, which represents an approximation for the movement of a liquid surface (see [7] for a detailed description), the boundary condition being $\nabla z(r=R) \widehat{n}=0$ for all $t$ and $\theta$. In the last situation, we describe an annular membrane [8,9], we have two fixed contours for this membrane, i.e. $z\left(r=r_{0}\right)=0$ and $z(r=R)=0$ for all $t$ and $\theta$. A practical situation similar to this is found in the design of a hornless moving-coil loudspeaker (see [10]).

Using the method of separation of variables equation (1) can be written in separable equations in the following way,

$$
\begin{aligned}
& \frac{\mathrm{d}^{2} T(t)}{\mathrm{d} t^{2}}+\gamma \frac{\mathrm{d} T(t)}{\mathrm{d} t}=\lambda_{1} c^{2} T(t), \\
& \frac{\mathrm{d}^{2} \Theta(\theta)}{\mathrm{d} \theta^{2}}=\lambda_{2} \Theta(\theta), \\
& \frac{\mathrm{d}^{2} R(r)}{\mathrm{d} r^{2}}+\frac{1}{r} \frac{\mathrm{d} R(r)}{\mathrm{d} r}+\left(\frac{\lambda_{2}}{r^{2}}-\lambda_{1}\right) R(r)=0,
\end{aligned}
$$

where $z(r, \theta, t)=T(t) \Theta(\theta) R(r)$. The general solution of these equations is given by

$$
\begin{aligned}
z(r, \theta, t)= & \sum_{m=0, n=1}^{\infty} \mathrm{e}^{-\gamma t / 2}\left(C_{1} J_{m}\left(k_{n}^{m} r\right)+C_{2} N_{m}\left(k_{n}^{m} r\right)\right)\left[\left(A_{n}^{m} \sin \omega_{n}^{m} t+\bar{A}_{n}^{m} \cos \omega_{n}^{m} t\right) \cos m \theta\right. \\
& \left.+\left(B_{n}^{m} \sin \omega_{n}^{m} t+\bar{B}_{n}^{m} \cos \omega_{n}^{m} t\right) \sin m \theta\right],
\end{aligned}
$$

where $\omega_{n}^{m}=\sqrt{c^{2}\left(k_{n}^{m}\right)^{2}+\gamma^{2} / 2}$ and $k_{n}^{m}$ depends on boundary conditions. Because of the damping the eigenfrequencies increase as expected.

For each boundary condition we have a particular solution for equation (5) we should analyse case by case. In the first case we set $C_{2}=0$, for the Neumann functions are not finite in $r=0$ and this solution makes no sense for the drum problem. In this case $k_{n}^{m}=\alpha_{n}^{m} / R$ where $\alpha_{n}^{m}$ are the zeros of the Bessel function

$$
J_{m}\left(\alpha_{n}^{m}\right)=0, \quad m=0,1,2, \ldots
$$

In the case in which the membrane is free to vibrate at its border we have $C_{2}=0$ too, using the same argument as above, and $k_{n}^{m}=\beta_{n}^{m} / R$, where $\beta_{n}^{m}$ are the zeros of the derivative of the Bessel function

$$
\frac{\mathrm{d} J_{m}\left(\beta_{n}^{m}\right)}{\mathrm{d} r}=0, \quad m=0,1,2, \ldots
$$

In the last case $C_{2} \neq 0$, because the solution of equation (5) does not pass through the point $r=0$ we have two equations to satisfy,

$$
\begin{aligned}
& C_{1} J_{m}\left(k_{n}^{m} R\right)+C_{2} N_{m}\left(k_{n}^{m} R\right)=0 \\
& C_{1} J_{m}\left(k_{n}^{m} r_{0}\right)+C_{2} N_{m}\left(k_{n}^{m} r_{0}\right)=0 .
\end{aligned}
$$

The nontrivial solutions are given by the transcendental equation [5]

$$
E\left(r_{0}, R, k_{n}^{m}\right)=N_{m}\left(k_{n}^{m} r_{0}\right) J_{m}\left(k_{n}^{m} R\right)-J_{m}\left(k_{n}^{m} r_{0}\right) N_{m}\left(k_{n}^{m} R\right)=0,
$$


where the constants satisfy the following relation:

$$
\frac{C_{2}}{C_{1}}=-\frac{J_{m}\left(k_{n}^{m} r_{0}\right)}{N_{m}\left(k_{n}^{m} r_{0}\right)}=-\frac{J_{m}\left(k_{n}^{m} R\right)}{N_{m}\left(k_{n}^{m} R\right)} .
$$

We can write equation (5) as a sum of normal modes (eigenmodes), which are functions that oscillate with the same eigenfrequency $\omega_{n}^{m}$. To illustrate the vibrations in normal modes let us assume in a particular case, the drum as initially at rest $z(r, \theta, 0)=0$. For this case $\bar{A}_{n}^{m}=\bar{B}_{n}^{m}=0$ and

$$
z(r, \theta, t)=\mathrm{e}^{-\gamma t / 2} \sum_{m=0, n=1}^{\infty} C_{1} J_{m}\left(k_{n}^{m} r\right) \times\left[A_{n}^{m} \cos m \theta+B_{n}^{m} \sin m \theta\right] \sin \omega_{n}^{m} t
$$

and the normal modes are

$$
R_{m, n}^{e}(r, \theta, t)=J_{m}\left(k_{n}^{m} r\right) \cos m \theta \sin \omega_{n}^{m} t \quad R_{m, n}^{o}(r, \theta, t)=J_{m}\left(k_{n}^{m} r\right) \sin m \theta \sin \omega_{n}^{m} t .
$$

We have two types of normal modes the odd $R_{m, n}^{o}$ and even $R_{m, n}^{e}$, which describe the movement of the circular membrane depending on the symmetry of our initial velocity, in this case. Finally we rewrite the general solution given by equation (5) as

$$
z(r, \theta, t)=\mathrm{e}^{-\gamma t / 2} \sum_{m=0, n=1}^{\infty}\left(a_{n}^{m} R_{m, n}^{e}+b_{n}^{m} R_{m, n}^{o}\right) \sin \omega_{n}^{m} t .
$$

\section{Computational details}

In this section we show how to solve, using the algebraic computational environment MAPLE, the model described in the last section, leading to the temporal evolution of the circular membrane in those situations. To explain the procedure we utilize as an example the more general case, the membrane with a hole at its centre. The first step to construct the temporal evolution series is to determine the coefficients $k_{n}^{m}$. To do this we plot equation (10) whose zeros we want to find, and look up the range in which the zero is located. So we can use the following MAPLE instructions to determine the exact position of the zeros. We present below the code to determine the zeros for $r_{0}=0.2, R=1$ and $m=0$ :

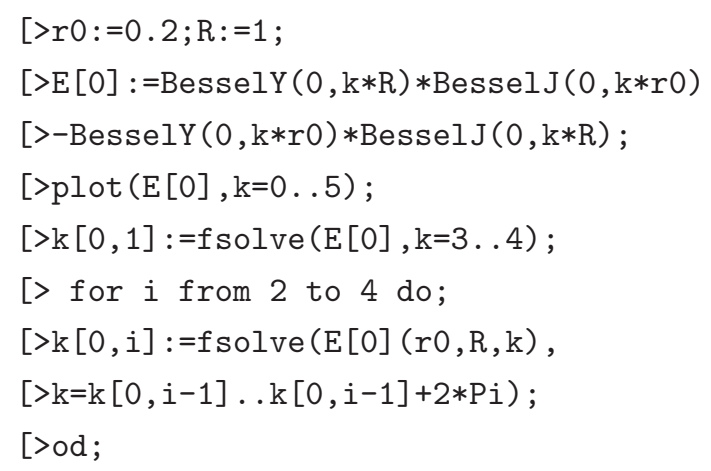

Plotting the expression for $E[0]$ we find the range where the first zero is localized and then we use the fsolve command to determine it. To find the other zeros we choose the range between the earlier zero and the earlier zero plus $2 \pi$, as the zeros of Bessel functions are spaced by a distance approximately close to $2 \pi$. Using the same procedure for other $m^{\prime}$, we find the zeros of equation (10). In the above case we just took the first four zeros. 


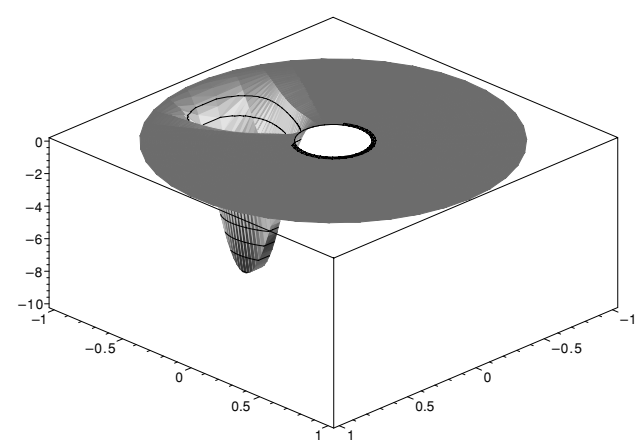

Figure 1. The $3 \mathrm{~d}$ graphic of initial velocity distribution, made with the plot $3 \mathrm{~d}$ command.

To determine the solution of equation (5) we need to define the initial situation of the membrane, that is, the initial form and velocity distribution of the membrane at some time. We choose the membrane initially at rest, $z(r, \theta, 0)=0$, giving it a kick at $t=0$ described mathematically by

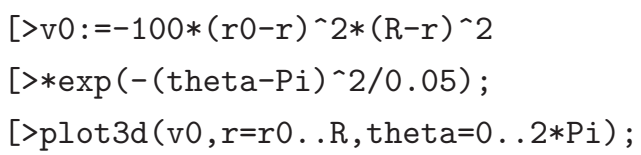

The graphic of the initial velocity distribution, using the plot3d command, is shown in figure 1.

For the initial velocity, a four-order polynomial in $r$ was used to calculate the integrals faster. Another interesting hint is to separate the angular and the radial dependences to facilitate the integral calculation which can be done as

$\left[>\mathrm{v} 0 \mathrm{r}:=(\mathrm{r} 0-\mathrm{r})^{\wedge} 2 *(\mathrm{R}-\mathrm{r})^{\wedge} 2\right.$;

$[>\mathrm{v} 0$ theta $:=\exp (-($ theta $-\mathrm{Pi}) \wedge 2 / 0.05)$;

Knowing the initial conditions we can calculate the temporal evolution of the membrane, using the following expressions:

$$
\left(\begin{array}{l}
A_{n}^{m} \\
B_{n}^{m}
\end{array}\right)=\frac{1}{\omega_{n}^{m}} \int_{0}^{\infty} \int_{0}^{2 \pi}\left(N_{m}\left(k_{n}^{m} r_{0}\right) J_{m}\left(k_{n}^{m} r\right)-J_{m}\left(k_{n}^{m} r_{0}\right) N_{m}\left(k_{n}^{m} r\right)\right) v_{0}(r, \theta) r\left(\begin{array}{c}
\cos m \theta \\
\sin m \theta
\end{array}\right) \mathrm{d} r \mathrm{~d} \theta .
$$

The above equation is obtained if we differentiate equation (5) with relation to time, evaluate in $t=0$ and compare with initial velocity distribution. As the Bessel functions form a complete set of eigenfunctions we can evaluate the coefficients $A_{m, n}$ and $B_{m, n}$. The coefficients obey $\bar{A}_{m, n}=\bar{B}_{m, n}=0$ because the initial shape is $z(r, \theta, 0)=0$.

For the radial function we choose in equation (5) the coefficients $C_{1}$ and $C_{2}$ according to equation (11) and evaluate a normalized function using the next commands:

[> for $m$ from 0 to 4 do;

[> for $\mathrm{n}$ from 1 to 4 do;

$[>\mathrm{R}[\mathrm{m}, \mathrm{n}]:=(\operatorname{BesselN}(\mathrm{m}, \mathrm{r} 0 * \mathrm{r}) * \operatorname{Bessel} \mathrm{J}(\mathrm{m}, \mathrm{k}[\mathrm{m}, \mathrm{n}] * \mathrm{r})$

$[>-\operatorname{BesselJ}(\mathrm{m}, \mathrm{r} 0 * \mathrm{r}) * \operatorname{BesselN}(\mathrm{m}, \mathrm{k}[\mathrm{m}, \mathrm{n}] * \mathrm{r})) / \mathrm{N}[\mathrm{m}, \mathrm{n}]$;

[>od; od; 
In the above equation, $N[m, n]$ is the normalization constant obtained by

[> for $i$ from 0 to 4 do;

[> for $j$ from 1 to 4 do;

$[>N[i, j]:=\operatorname{int}((R[i, j]) \sim 2 * r, r=r 0 . . R)$;

[>od; od;

We first evaluate the angular then the radial integrals, as follows:

[> for $m$ from 0 to 4 do;

[>ange $[\mathrm{m}]:=$ int $(\mathrm{v} 0$ theta $* \cos (\mathrm{m} * \mathrm{p})$, theta $=0 . .2 * \mathrm{Pi})$;

[>ango $[\mathrm{m}]:=$ int (v0theta $* \sin (\mathrm{m} * \mathrm{p})$, theta $=0 . .2 * \mathrm{Pi})$;

[>od;

[> for m from 0 to 4 do;

[> for $\mathrm{n}$ from 1 to 4 do;

$[>\mathrm{A}[\mathrm{m}, \mathrm{n}]:=$ ange $[\mathrm{m}] * \operatorname{int}(\mathrm{v} 0 \mathrm{r} * \mathrm{R}[\mathrm{m}, \mathrm{n}] * \mathrm{r}, \mathrm{r}=\mathrm{r} 0 \ldots \mathrm{R})$;

$[>\mathrm{B}[\mathrm{m}, \mathrm{n}]:=$ ango $[\mathrm{m}] * \operatorname{int}(\mathrm{vOr} * \mathrm{R}[\mathrm{m}, \mathrm{n}] * \mathrm{r}, \mathrm{r}=\mathrm{r} 0 \ldots \mathrm{R})$;

$[>$ od;

$[>$ od;

Now we finally sum up the coefficients to determine the temporal evolution of the circular membrane. In MAPLE we can evaluate it using these following commands:

[> for $\mathrm{m}$ from 0 to 4 do;

[> for $\mathrm{n}$ from 1 to 4 do;

$[>$ gamma $:=2$;

$[>$ omega $[\mathrm{m}, \mathrm{n}]:=\operatorname{sqrt}(\mathrm{k}[\mathrm{m}, \mathrm{n}] \wedge 2+\operatorname{gamma} \wedge 2 / 2)$;

$[>\mathrm{ze}[\mathrm{m}, \mathrm{n}]:=\mathrm{A}[\mathrm{m}, \mathrm{n}] * \mathrm{R}[\mathrm{m}, \mathrm{n}] * \sin ($ omega $[\mathrm{m}, \mathrm{n}] * \mathrm{t}$ )

$[>* \exp (-$ gamma*t/2)*cos (m*theta) ;

$[>\mathrm{zo}[\mathrm{m}, \mathrm{n}]:=\mathrm{B}[\mathrm{m}, \mathrm{n}] * \mathrm{R}[\mathrm{m}, \mathrm{n}] * \sin ($ omega $[\mathrm{m}, \mathrm{n}] * \mathrm{t}$ )

$[>* \exp (-$ gamma*t/2)*sin (m*theta); [> od;

$[>\mathrm{z}:=\operatorname{sum}(\operatorname{sum}(\operatorname{Re}(\mathrm{ze}[\mathrm{m}, \mathrm{n}])+\operatorname{Re}(\mathrm{zo}[\mathrm{m}, \mathrm{n}]), \mathrm{n}=1 \ldots 4)$

$[>, \mathrm{m}=0 . .4)$;

In the above calculation we extracted the real part of ZE and ZO, because when MAPLE gives the coefficients of the expansions $A[m, n]$ and $B[m, n]$ it keeps an imaginary part, which is actually zero. We have adopted the propagation velocity $c=1$ and the damping constant $\gamma=2$.

The procedure for the other two cases can be done if we change the calculation of $k[m, n]$ and $R[m, n]$ according to the description in section 2 .

\section{Graphics}

\subsection{The normal modes of vibration}

The temporal evolution which determines the position of the membrane in time is a superposition of normal modes of the membrane with the corresponding boundary conditions. 

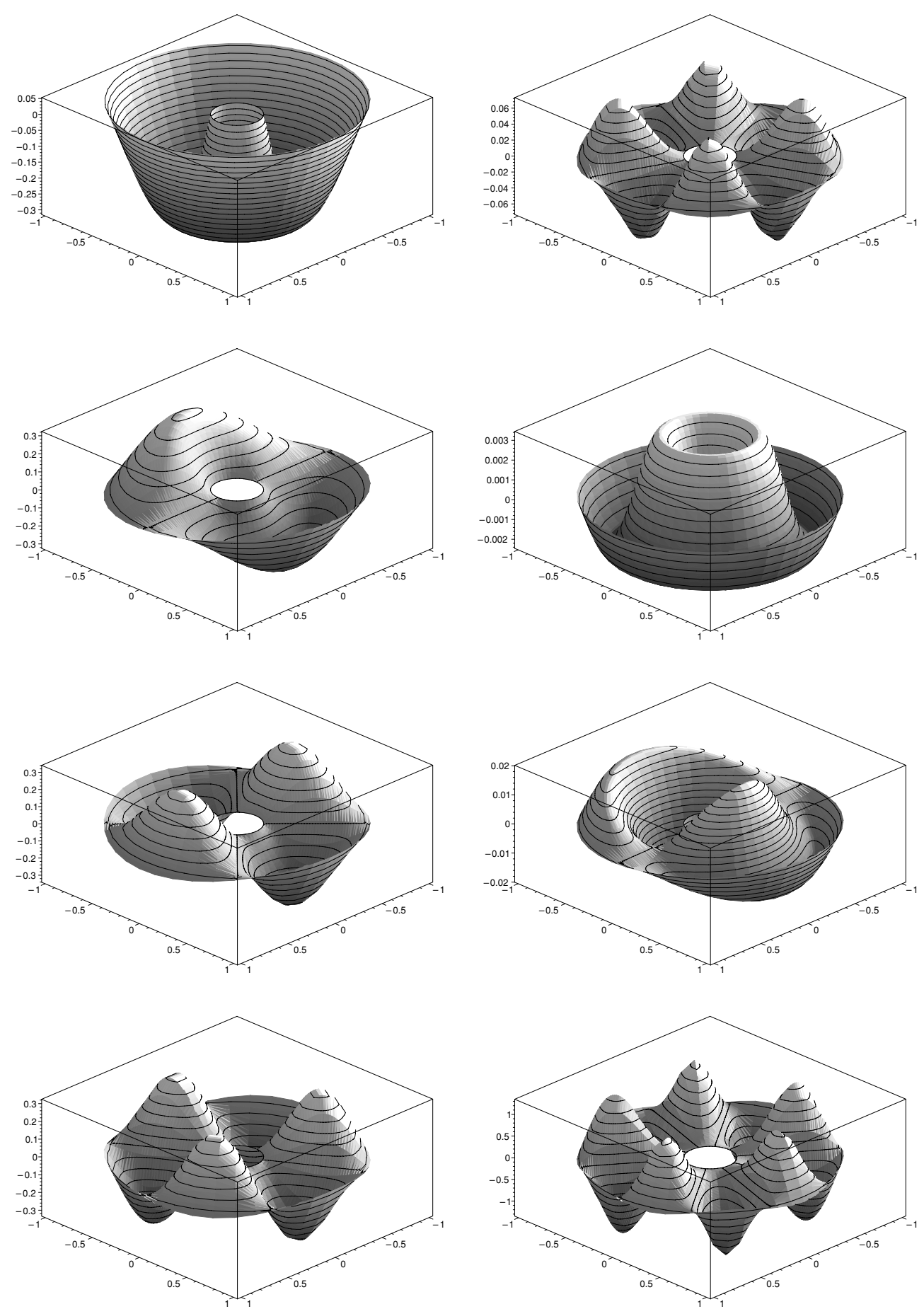

Figure 2. Graphics of the first eight pure harmonics for the membrane with fixed contour and a central hole, in the left panel we have the first four harmonics and in the right panel the next four, $t=0.2$. 

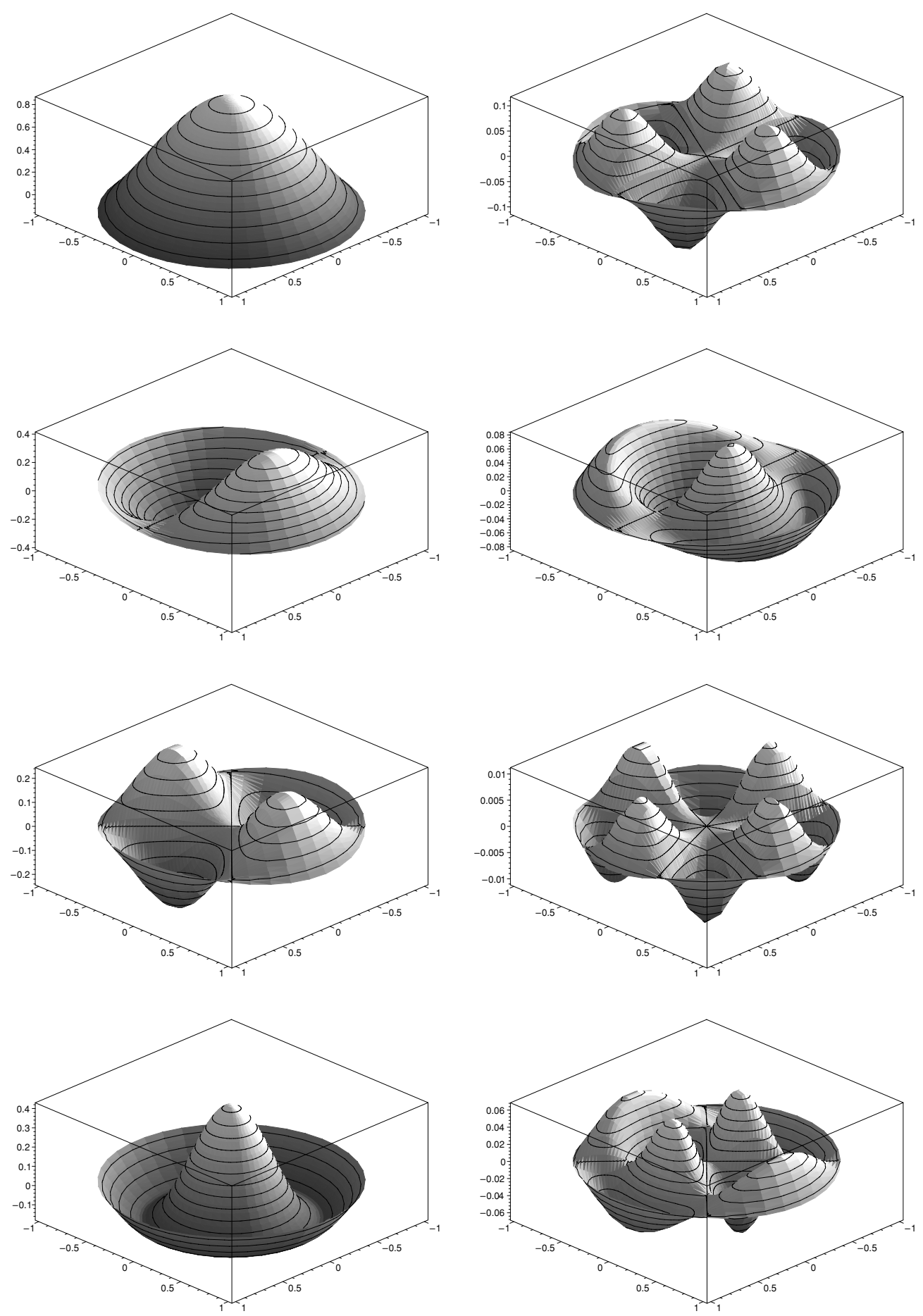

Figure 3. Graphics of the first eight pure harmonics for the membrane with fixed contour, in the left panel we have the first four harmonics and in the right panel the next four, $t=0.2$. 

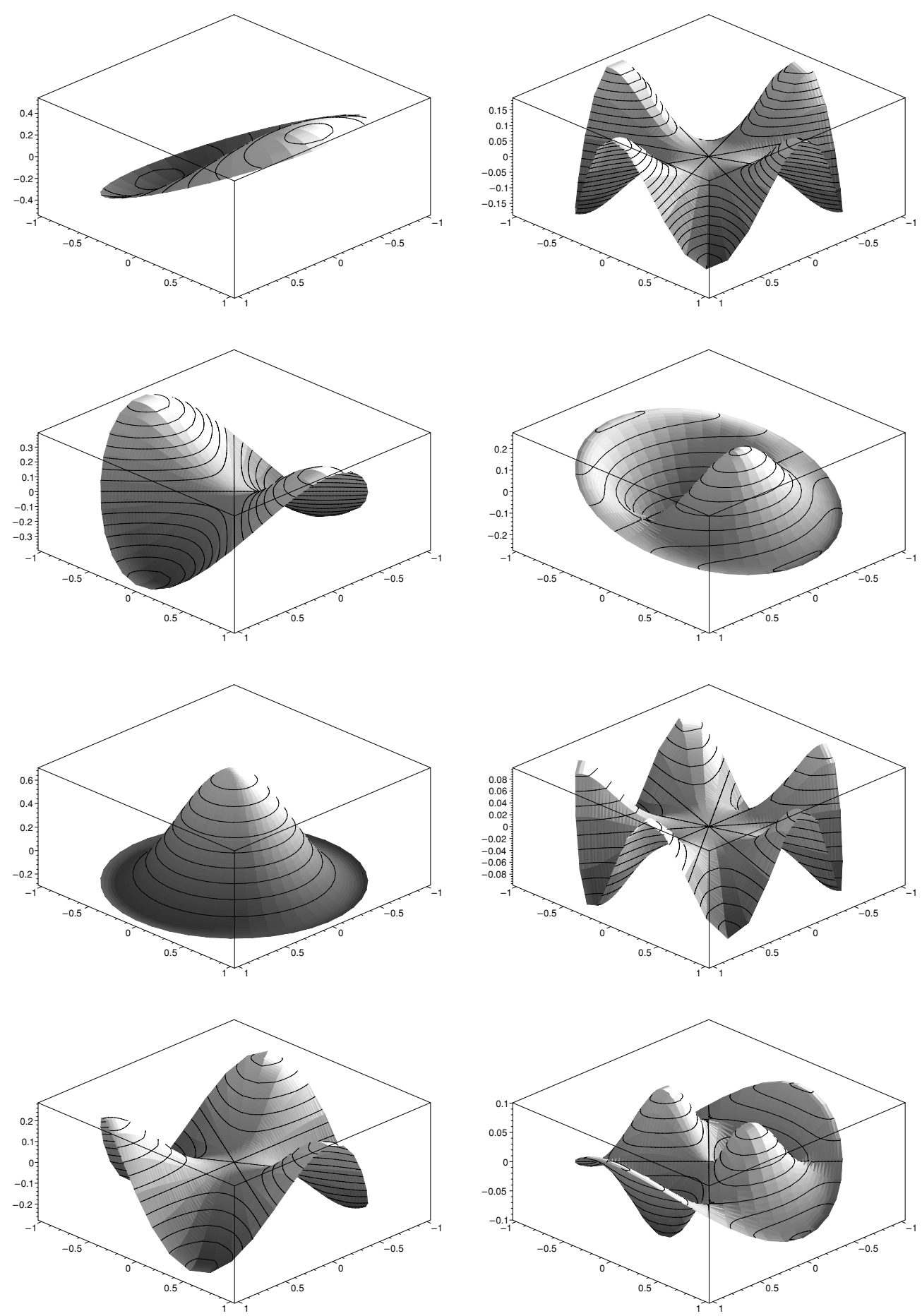

Figure 4. Graphics of the first eight pure harmonics for the membrane with a free contour, in the left panel we have the first four harmonics and in the right panel the next four, $t=0.2$. 

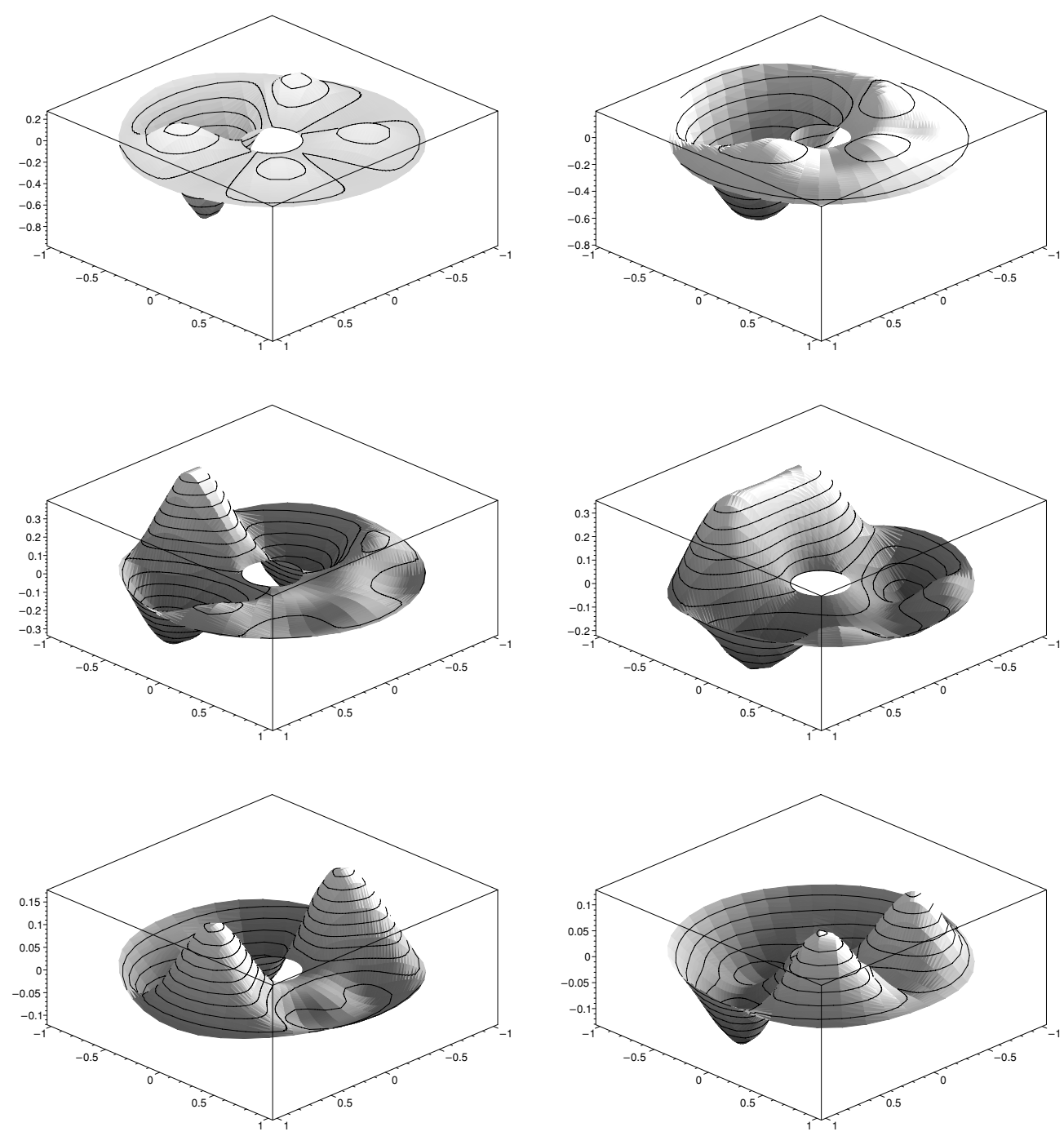

Figure 5. Graphics of a membrane with a hole for different times, in the left panel we have $t=$ $0.1, t=0.7$ and $t=1.5$ and in the right panel we have $t=0.4, t=1.0$ and $t=1.8$.

The normal modes are ordered according to the eigenfrequencies, which means that the fundamental mode is correspondingly the smallest eigenfrequency, the first excited normal mode is correspondingly the next smallest eigenfrequency and so on.

In this section we explore these modes graphically using tridimensional plots that can be visualized by the MAPLE commands below:

$[>t:=0.2 ; \quad[>$ with $($ plots $) ;$

$[>\mathrm{m}:=0 ; \mathrm{n}:=1$;

$[>\mathrm{Ro}[\mathrm{m}, \mathrm{n}]:=\mathrm{R}[\mathrm{m}, \mathrm{n}] * \cos (0 *$ theta $) * \sin ($ omega $[\mathrm{m}, \mathrm{n}] * \mathrm{t})$;

$[>\operatorname{plot} 3 \mathrm{~d}([\mathrm{r} * \cos ($ theta $), r * \sin ($ theta $), \operatorname{Ro}[\mathrm{m}, \mathrm{n}]]$

$[>, r=r 0 . . R$, theta $=0 . .2 * P i)$; 

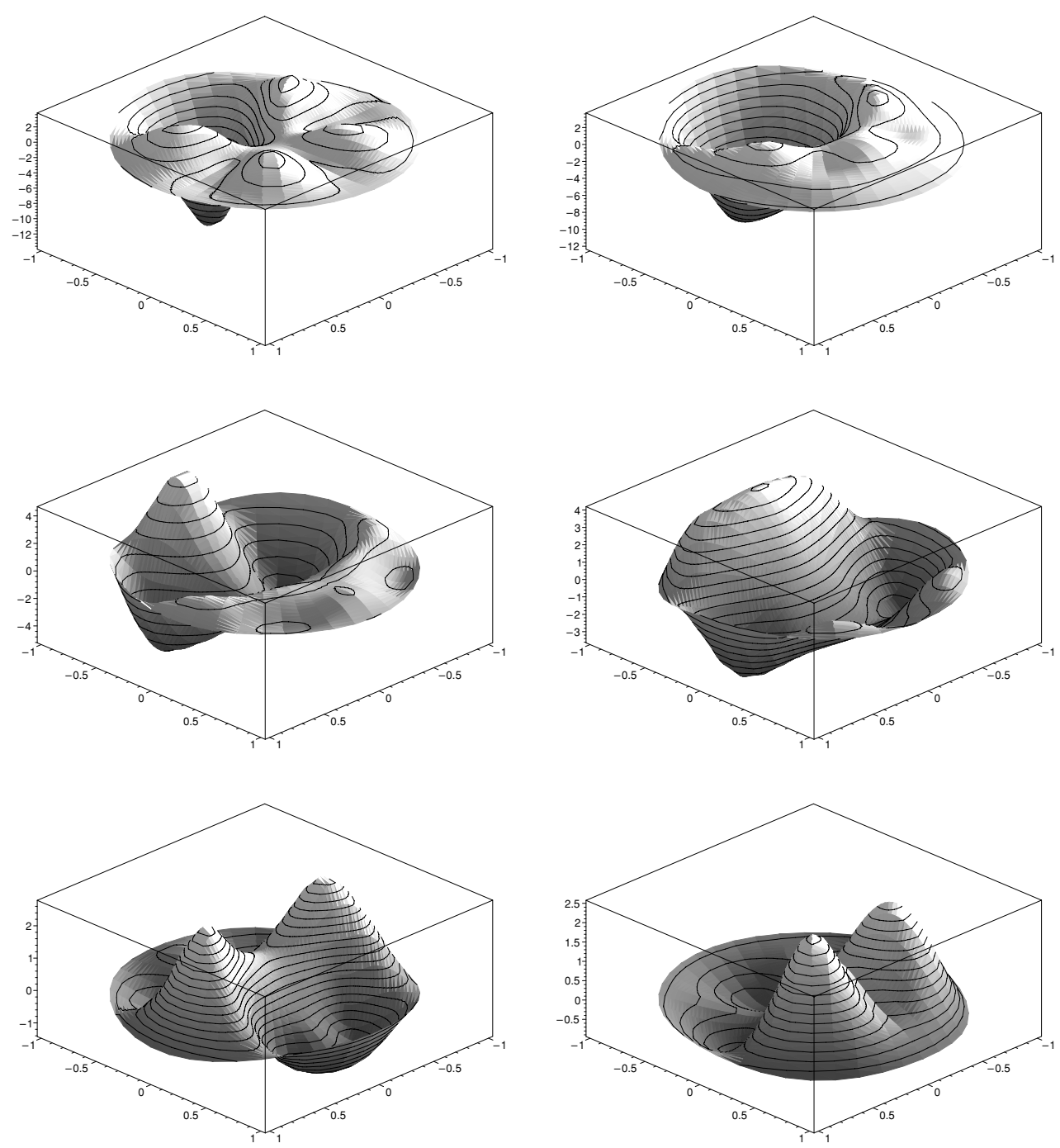

Figure 6. Graphics of a fixed membrane at different times, in the left panel we have $t=0.1, t=0.7$ and $t=1.5$ and in the right panel we have $t=0.4, t=1.0$ and $t=1.8$.

The instructions above just plot the fundamental normal mode at time $t=0.2$, to visualize the other normal modes we just change the index $(m, n)$ according to their eigenfrequencies. We show the normal modes of the circular membrane with a hole at the centre in figure 2 , the circular membrane in figure 3 and the liquid membrane in figure 4. The temporal evolution of the normal modes can be done using the following sequence:

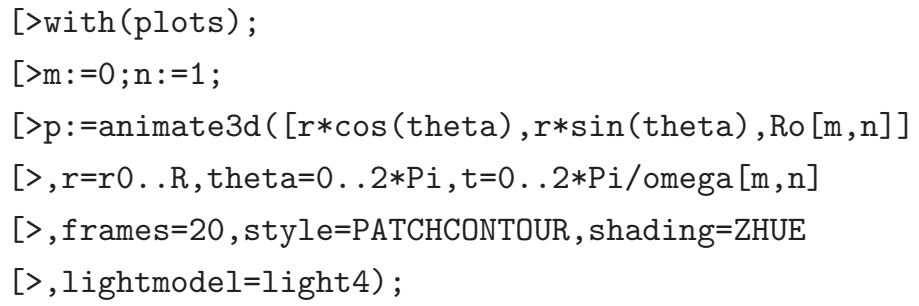



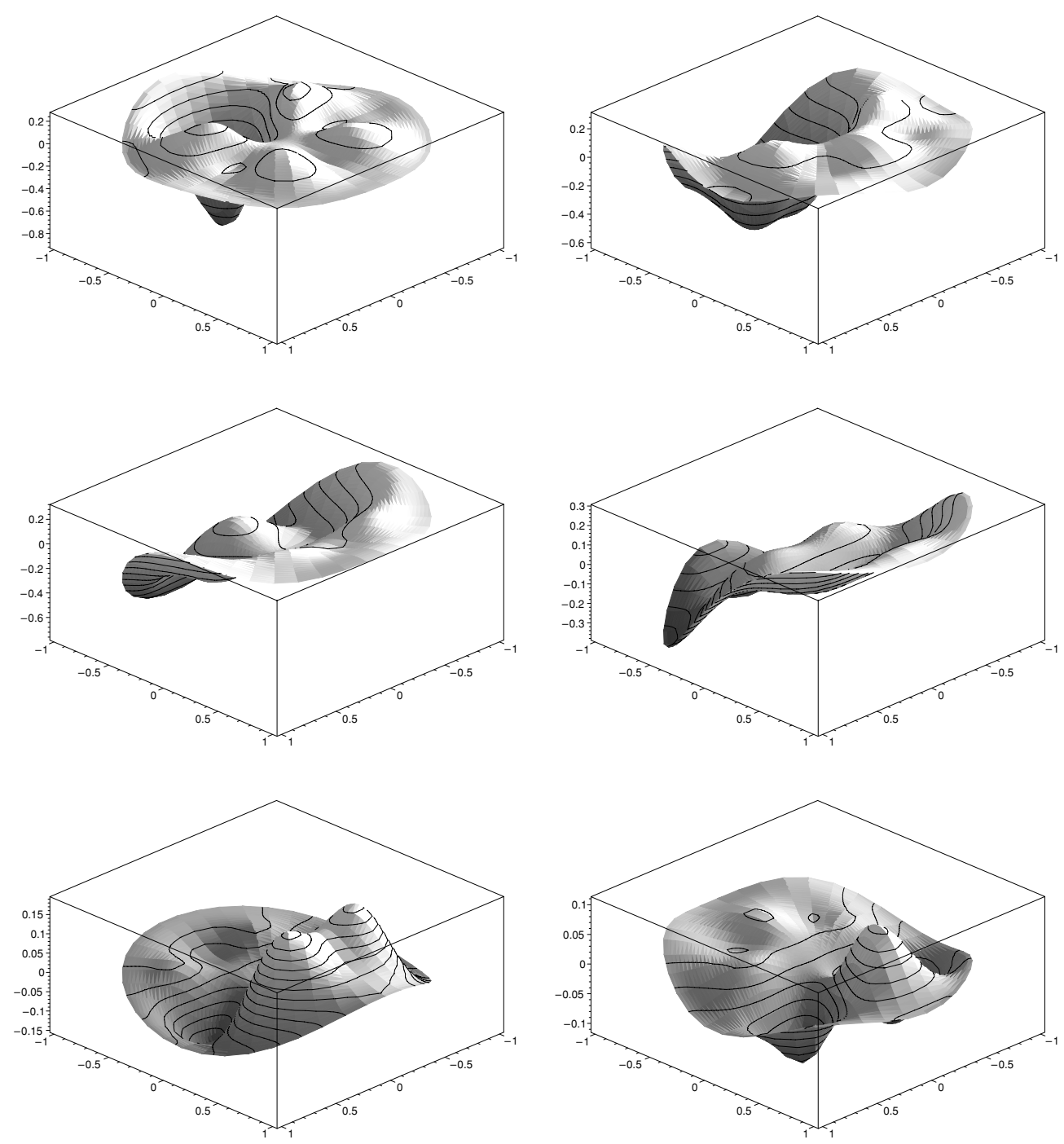

Figure 7. Graphics of a membrane with a free contour for different times, in the left panel we have $t=0.1, t=0.7$ and $t=1.5$ and in the right panel we have $t=0.4, t=1.0$ and $t=1.8$.

The command with(plots) loads the plots package where the animate $3 d$ routine is localized, which generates an animation so the reader can visualize the simulation on his own computer.

\subsection{Temporal evolution}

In this section we present instructions to illustrate the temporal evolution, the final results of these simulation which are shown in figures 5-7. To produce a single figure we fix a time and use the plot $3 d$ command as follows:

$[>\mathrm{t}:=0.1$;

$[>\mathrm{plot} 3 \mathrm{~d}([\mathrm{r} * \cos ($ theta $), r * \sin ($ theta $), z]$

$[>, r=r 0 . . R$, theta $=0 . .2 * P i)$; 
To create a graphical animation of these temporal evolutions we use the sequence of commands presented below:

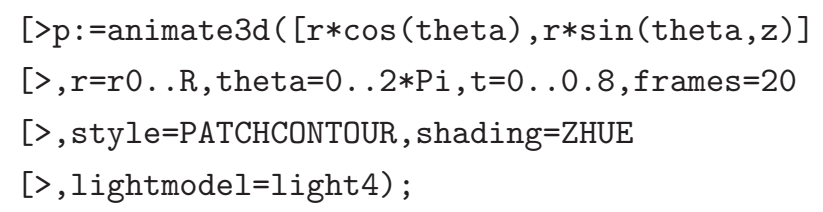

The animation created by the above commands can be visualized in the mathematical physics course home page located at http://basalto.if.sc.usp.br/cursos/FISMAT/ensaios.html.

\section{Conclusions}

In this paper we have presented a survey analysis of a graphically challenging physical problem, that of a membrane vibrating under three typical situations of constraint. The procedural steps to generate these graphical sequences are, despite some tedious calculations which are actually done by the computer, simple. The result is encouraging. While in printed form we can only check the shape of the membrane which is made of a superposition of eigenmodes (a grace of linear systems), to produce an animated version of this sequence is a straightforward step. One can have, by looking at the actual animation movie, an immediate feeling of the response in time of the membrane as kinetic energy flows from the point of the initial hit to the border under a specific constraint and back with simultaneous degradation of this energy.

The use of an algebraic computer environment, as presented in this work, is suggested as a complement to the formal teaching methods. The analytical properties of special functions have yet to be mastered by the student, but the results of such eigenmode analysis ought not to be as dry as just an equation describing its points at different times.

Other very interesting physical problems can be visualized in this manner, such as the vibration of porous balls or even simple studies of waves in strings. Work to conclude a case study in these directions is being carried out.

\section{Acknowledgment}

The authors are grateful to Fapesp (processes: 02/02504 and 01/14572-9) for financial support.

\section{References}

[1] Euler L 1764 De motu vibratorio tympanorum Novi. Comm. Acad. Petrop. 10 243-60 (published in 1766)

[2] Watson G N 1966 A Treatise of the Theory of Bessel Functions (Cambridge: Cambridge University Press)

[3] Bessel F W 1824 Untersuchung des Theils der planetarischen Störungen welcher aus der Bewegung der Sonne entsteht Berliner Abh. 1-52 (published in 1826)

[4] Portugal R, Golebiowski L and Frenkel D 1999 Oscillation of membranes using computer algebra Am. J. Phys. 67534

[5] Butkov E 1968 Mathematical Physics (Reading, MA: Addison-Wesley)

[6] Mary Boas L 1961 Mathematical Methods in the Physical Sciences (New York: Wiley)

[7] Mary Lamb H 1945 Hydrodynamics 6th edn (New York: Dover) chapter VIII, pp 282, 284

[8] McLachlan N W 1941 Bessel Functions for Engineers (Oxford: Oxford University Press)

[9] McLachlan N W 1951 Theory of Vibrations (New York: Dover)

[10] McLachlan N W 1934 Loud Speakers (Oxford: Claredon) 